\title{
The Serotonergic System in Diet, Obesity and Mental Health
}

\author{
Lina Begdache ${ }^{1 *}$ and Jade Marhaba ${ }^{2}$ \\ ${ }^{1}$ Department of Health and Wellness Studies, Binghamton University, USA \\ ${ }^{2}$ Upstate Medical School, State University of New York, USA \\ *Corresponding author: Lina Begdache, Department of Health and Wellness Studies, Binghamton University, US

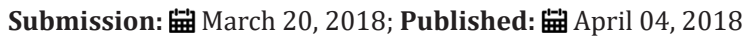

\begin{abstract}
Mental health and obesity research seldom intersect despite that poor diet and serotonergic system disturbances are common in both etiologies. Many interplaying factors lead to a concomitant dysregulation of the feeding centers and the limbic emotional processing centers. Leptin resistance, poor diet and lifestyle, inflammation, and psychological stress Interwine to disrupt these functions. Despite that many neurotransmitters play a role in these processes; the serotonergic system appears to be at the heart of these disturbances. Therefore, shedding the light on these factors and their effect on the serotonergic system may support a comprehensive therapeutic approach. Another goal for this review was to evaluate the cellular and molecular derangements associated with feeding disturbances and mental health to better shed the light on the intricate factors leading to their development.
\end{abstract}

Keywords: Leptin; Serotonin; Diet; Exercise; Inflammation; Stress; Obesity; Anxiety; Depression

Abbreviations: BBB: Blood-Brain Barrier; IL: Interleukin; 5-HT: 5-Hydroxytryptamine; HPA: Hypothalamic Pituitary Adrenal; PVN: Paraventricular Nucleus of the Hypothalamus; Tph: Tryptophan Hydroxylase; KO: Knockout; CNS: Central Nervous System; GABAergic: Gamma Aminobutyricacid Ergic; LNAA: Large Neutral Amino Acids; EPA: Eicosapentaenoic Acid; DHA: Docosahexaenoic Acid; CV: Cardiovascular; NPY: Neuropeptide Y; AgRP: Agouti Related Protein; POMC: Proopiomelanocortin; CART: Cocaine and Amphetamine Regulated Transcript; NMDA: N-methyl-D-aspartate

\section{Introduction}

The fields of mental health and obesity research are less likely to interconnect despite that both conditions have poor diet and serotonergic system disturbances as a common etiology. Obesity is usually linked to dysregulation of the hypothalamic neural circuits of the feeding centers. Mental distress, namely anxiety and depression, appear to be linked to dysregulation of neural circuits in the limbic regions such as the hippocampus and the amygdala. However, neural circuits involved in both morbidities are activated by the same set of neurotransmitters. Monoamines such as dopamine, norepinephrine and serotonin are the major target for psychiatric drugs. The same neurotransmitters are also the target of some weight loss medications that regulate the hypothalamic feeding centers. Although dopamine, and to a lesser extent norepinephrine, have been reported to have some effect on the feeding center, their main role is in the limbic reward system. On the other hand, serotonin appears to play a key role in the dysregulation of both systems. Additionally, obesity and mental distress have been both associated with increased risk for dementia and cognitive decline [1]. Poor diet increases systemic inflammation which contributes to the etiology of cognitive impairment. [2] Consequently, the brain becomes susceptible to oxidative stress due to the limited endogenous antioxidant enzymes and the tight regulation over transport of antioxidants and others across the blood-brain barrier (BBB). Inflammation in the brain has been linked to leptin resistance [3] and to mental distress [4,5]. Patients with mental disease and those with a body mass index within obese ranges commonly have activated inflammatory pathways manifested by increased pro-inflammatory cytokines such as interleukin (IL)-6, and/or C-reactive protein as positive markers of acute-phase proteins $[4,6]$. Inflammation and subsequent oxidative stress disturb homeostasis by altering redox signaling and gene expression [7].

The purpose of this review was to elucidate the different components of the serotonergic systems that control the limbic and the feeding centers. Another goal was to evaluate the cellular and molecular derangements associated with feeding disturbances and mental health to better shed the light on the intricate factors leading to their development.

\section{Discussion}

The serotonergic system: the interface between feeding behavior and mental health

Serotonin, or 5-hydroxytryptamine (5-HT), and its receptors play a vital role in the regulation of nearly all brain functions despite 
that the majority of serotonin is produced in the gut [8]. However many of the serotonergic neuronal projections within the cortical, limbic, midbrain, and hindbrain regions appear to originate from the brainstem [9]. There are currently fifteen identified serotonin receptors which are grouped into seven families based on their intracellular signaling mechanisms [10]. In addition, all brain regions express several serotonin receptors with typically specific subtype patterns [11]. However, individual neurons also express different serotonin receptors with disparate effects to modulate neuronal activity [12].

Serotonin regulates brain regions responsible for emotional processing (which include the prefrontal cortex and the limbic system) and the hypothalamic feeding centers such as the hypothalamus, the extended amygdala and brainstem $[13,14]$. In fact, dysregulation of the serotonergic system in these brain areas has been implicated in the pathogenesis of many psychiatric conditions and disordered eating $[15,16]$. Alternatively, chemicals that promote the increase in 5-HT neurotransmission are typically prescribed for the treatment of depression, anxiety, and in some instances obesity. At night (or in dim light), serotonin is metabolized further into melatonin in the pineal gland. Therefore, 5-HT also contributes to the circadian rhythm. A mutual functional interface exists between central 5-HT system and the hypothalamicpituitary-adrenal (HPA) axis under normal physiological conditions. Therefore, serotonin plays a significant role in the HPA axis regulation to turn off centrally the stress response through a negative feedback loop [17]. In fact, the hippocampus and the paraventricular nucleus of the hypothalamus (PVN), part of the limbic serotonergic system, are regions in which components of the HPA axis and the 5-HT systems interact extensively. Multiple central and peripheral inputs converge on PVN to modulate the HPA axis where corticotropin-releasing hormone is synthesized. Subsequently, adrenocorticotropin signals the pituitary gland to release cortisol from the adrenal cortex [18]. The steady rise in plasma cortisol concentration attenuates the stress response in the hypothalamus and pituitary gland via a negative feedback loop. Consequently, dysregulation of HPA and 5-HT systems contributes to the pathophysiology of mental diseases. Therefore, dietary or pharmacological therapies need to address this dysregulation to produce a healing effect [19].

\section{Tph2 and brain serotonin synthesis}

Serotonin is synthesized in the brain and in the peripheral tissue by a rate-limiting enzyme: tryptophan hydroxylase (Tph). Tph exists as two isoforms, Tph1 and Tph2, which are encoded by two separate genes. Tph1 is the rate-limiting enzyme that controls 5-HT synthesis in the periphery (gut, pineal gland, spleen and thymus); whereas Tph2 is more localized to the brain [20]. In humans, Tph2 is highly expressed in frontal cortex, thalamus and hypothalamus and less expressed in the hippocampus and amygdala, and hardly detectable in peripheral tissues [21]. Evidence from the literature reveals that Tph2 gene expression is highly inducible and is closely related to the stress response. However, glucocorticoids effect on Tph2 expression appears to be species-dependent and may be tissue-specific [22]. Additionally, daily fluctuations in glucocorticoids levels modulate the circadian rhythmic Tph2 expression, which explains the reduction in brain serotonin with sleep deprivation. This phenomenon was demonstrated through adrenalectomy in lab animals which led to a total suppression of the daily rhythmic Tph2 mRNA expression in the raphe nucleus. In contrary, glucocorticoid restoration in the same models reinstated Tph2 expression [23]. In fact, serotonin-receptor knockout (KO) studies reflect these critical functions of 5-HT in different organ systems such as the central nervous system (CNS), GI, heart, endocrine functions and others. Autonomic functions such as sleep, respiration, body temperature, blood pressure and heart rate are severely altered with disrupted Tph2 expression or absence of 5-HT [24]. To better understand the effect of Tph2 gene expression in relation to 5 -HT functions, $\mathrm{KO}$ mice studies completely lacking 5 -HT in the brain, reported growth retardation and increased lethality in early life stages. These findings suggest that Tph2 gene expression is closely associated with the biological clock and the stress response. Additionally, sleep quality may affect levels of Tph2 expression and thus 5-HT concentrations.

\section{Function of 5-HT2C receptors}

CNS serotonergic neurons exert a moderating effect on an extensive neural network through modulation of the gammaaminobutyric acid-ergic (GABAergic) neurons activity. This extended control explains the widespread effect of serotonin on human behavior [11]. These behaviors include mood, feeding and appetite, reward, aggression, perception, memory, sexuality, anger and attention, among others. Although the neural circuits responsible for these behaviors have not been all elucidated, it appears that different brain regions control specific behaviors via an explicit concoction of serotonin receptor subtypes. 5-HT2C expression is particularly high in the limbic system which implies its fundamental role in mental distress and disordered feeding. In fact, 5-HT2C receptors KO in mice studies induce concomitant hyperphagia [25], and anxious behaviors [26]. Additionally, 5-HT2C receptor regulates reward processing, locomotion and energy balance [27]. Weight loss and psychiatric medications are often associated with several side effects and in most instances; they exhibit an opposite effect on the other co-morbidity. For instance, weight loss medications increase the incidence of mental distress, and several psychiatric medications promote weight gain. Therefore, therapies that support treatment of both conditions simultaneously are warranted. Taken all evidence collectively, Tph2derived 5-HT multitasks between behavior regulation, autonomic control and modulation of metabolic pathways which implicates it in the pathophysiology and pharmacology of mental disease.

\section{Effect of diet and exercise on serotonin}

Dietary composition and exercise have a significant effect on serotonin synthesis. The essential amino acid tryptophan is the precursor for 5-HT production. Tryptophan shares with the other large neutral amino acids (LNAAs) a common carrier across BBB. Therefore, tryptophan/LNAA ratio determines the amount of tryptophan supplied to the brain. A protein-rich diet provides 
copious endogenous amounts of amino acids which lower the tryptophan/LNAA ratio. Subsequently, serotonin production decreases. On the other hand, a complex carbohydrate-rich diet induces a steady rise in serum insulin, which promotes a continuous amino acid uptake by peripheral tissue. Consequently, tryptophan/ LNAA ratio increases which leads to a constant rise in central 5-HT synthesis [28]. This explains the reason behind increased carbohydrate cravings with a negative mood. Therefore, dietary interventions to promote mental wellbeing and weight control need to assess the ratio of protein to carbohydrates as well as the quality of carbohydrates consumed.

Inflammation induces leptin resistance which has been linked to obesity and mental distress $[3,29,30]$. A healthy ratio of essential fatty acids omega- $6 / 3$ is needed to prevent low chronic inflammation. In fact, a high ratio of omega $6 / 3$ has been linked to mental distress severity and obesity $[31,32]$. Dietary omega- 6 fats are precursors for arachidonic acid from which pro-inflammatory prostaglandins are derived. Marine omega-3 fats (eicosapentaenoic acid (EPA eicosapentaenoic acid) exhibit an anti-inflammatory effect through production of anti-inflammatory prostaglandins. Increase in cortisol levels associate with reduced levels of EPA [33], which add to the inflammatory response. Another important omega-3 fat is docosahexaenoic acid (DHA), which is one of the most abundant fats in the brain. Low levels of DHA have also been linked to mental distress in animal and human models $[34,35]$. Therefore, a balance between omega- 6 and omega- 3 fats is needed to prevent chronic low-grade inflammation and improve mood as well as leptin sensitivity [36,37].

Exercise promotes muscle use of branched-chain amino acids (BCAA), a type of LNAA, which reduces the competitive transport of tryptophan to the brain [38,39]. In fact, brain stem and hypothalamus synthesis of 5-HT significantly increases with acute cardiovascular (CV) exercise. It appears that serotonin synthesis in the cerebral cortex and hippocampus remains low with acute CV exercise. However, chronic CV exercise induces sustainable adaptation that leads to increased synthesis and metabolism of 5-HT in the cerebral cortex and in the hippocampus [39]. Therefore, the antidepressant effect of CV exercise typically comes from chronic exercise and not much from occasional activities [40]. CV exercise also sensitizes the muscle to efficiently oxidize glucose for energy which reduces inflammation and improves mood as well as body weight.

\section{Serotonin and the gastrointestinal system}

Discussion on feeding behaviors cannot be complete without addressing the gut hormones. The gut is the largest endocrine organ in the human body which contributes numerous peptide hormones critical for the short-term feeding behaviors. These hormones mainly control meal initiation and termination. The key peptides include cholecystokinin, ghrelin, pancreatic polypeptide, and others [41]. All gut hormones have an anorexic effect except for ghrelin, which increases hunger and promotes regulation of energy metabolism. In fact, ghrelin is an endogenous ligand for the growth hormone secretagogue receptors primarily found in the pituitary gland, on hypothalamic neurons in the brainstem and recently reported in immune cells $[42,43]$. Additionally, the growth hormone is of importance for maintaining synaptic plasticity, especially with sleep deprivation [44]. Therefore, during stressful times, when sleep quality declines, ghrelin may have a protective effect on the neural circuit integrity involved in emotional processing and feeding centers. Additionally, ghrelin acts on the innate and adaptive immune systems to suppress inflammation and induce an anti-inflammatory profile. However, ghrelin actions differ between peripheral tissue and the brain. Peripheral daily administration of ghrelin in lab animals induced weight gain through reduced fat utilization. However, intra-cerebroventricular administration of ghrelin in the same experimental models led to a dose-dependent increase in food intake and body weight. Moreover, ghrelin seems to have an anxiolytic effect. In animal studies, ghrelin administration attenuates anxious behavior through the modulation of the HPA axis [45]. Like serotonin, ghrelin modulates HPA axis by integrating central circuitry involved in anxiety and psychological stress. In human models, high cortisol levels lead to higher production of ghrelin to support the negative feedback loop described earlier. Like serotonin, circulating ghrelin levels are also reduced in patients with mental distress. Taken all together, ghrelin is at the juncture between feeding, growth, energy metabolism and stress response [46]. Based on the given facts, experiencing intermittent low dose ghrelin secretion may have a positive effect on stress and mood. However, higher ghrelin levels may induce an increase in feeding behavior and body weight [47]

\section{Feeding Centers}

Feeding behaviors are regulated by a complex network that covers different areas of the brain. However, the hypothalamus is the main regulatory component of this network. The arcuate nucleus of the hypothalamus houses two major types of neurons that regulate feeding behavior. The orexigenic neurons neuropeptide Y (NPY)/ agouti-related protein (AgRP) promote feeding upon stimulation. The anorexigenic proopiomelanocortin (POMC) and cocaine-andamphetamine-regulated transcript (CART) peptides inhibit feeding when activated [48]. Both systems work through the cAMP second messenger system. Additionally, the two systems project fibers to different areas of the brain that control feeding behavior. NPY/ AgRP neuronal projection extends to POMC neurons acting as an inverse agonist to stimulate feeding.

\section{Leptin}

Leptin, a hormone produced by the adipose tissue, is a longterm regulator of feeding behavior. The 16-kDa hormone enters the brain through the cerebrospinal fluid at low nanomolar concentrations (10-20 nM) [49]. Leptin mRNA has been localized in neuronal populations including the hippocampus [50], which suggests confined production as well. Leptin is believed to be part of the set-point theory which describes an increase in food intake and a decrease in energy expenditure with a decrease in serum leptin levels and vice-versa [51,52]. Leptin exerts its effect on hypothalamic as well as brainstem serotonergic neurons which proposes that these regions are closely associated with regulation of food intake [53]. Precisely, abundant leptin receptors exist in 
the hypothalamic orexigenic neurons NPY/AgRP as well in the anorexigenic POMC and CART peptides [48]. AgRP neurons have direct inhibitory effects on POMC neurons; however, a reciprocal synapse from POMC neurons to AgRP/NPY neurons does not exist [54]. Therefore, this dual presence of leptin receptors in the microcircuit is crucial for modulation of the feeding response. The integration of peripheral and central inputs produces an integrated response necessary for the inhibition of NPY/AgRP neurons and stimulation of POMC/CART neurons [55]. The leptin-dependent inhibition of AgRP/NPY neurons is believed to involve modulation of the voltage-gated $\mathrm{K}+$ channels. Diet-induced obesity in animal models has been associated with continuous activation of NPY neurons due to inability of leptin to hyperpolarize the resting membrane potential.

\section{Psychological stress and leptin levels}

Animal and human studies suggest that leptin and stress interact though a closed loop. Low leptin levels associate with increased symptoms of depression in young females, independent of body fat or weight [30]. Additionally, those with clinical depression exhibit lower levels of leptin after controlling for fat mass or body weight. Leptin acts as an anti-stress hormone by attenuating the response of the adrenocorticotropic and corticosterone hormones to stress and by inhibiting glucocorticoid synthesis and its release from the adrenal cortical cells [56]. Acute stress does not affect leptin levels in lab animals. However, chronic stressors decrease basal plasma levels of leptin independent of body weight [57] Remarkably, previous negative life experiences sensitize leptin secretion response to acute stress. Reduced serum leptin levels with psychological stress correlate with behavioral alterations indicative of mental distress. Mechanistically, leptin regulates gene expression in the limbic brain areas, particularly the hippocampus, which is implicated in the pathophysiology of major depressive disorders [57]. Direct activation of the leptin receptor in the hippocampus but not in the hypothalamus produces antidepressant-like behavioral effects in experimental models, suggesting that the hippocampus is a neuroanatomical substrate for leptin's antidepressant-like actions. Therefore, as part of a therapeutic approach, an initial stress reduction is needed to support the anti-stress effect of leptin to consequently improve mood and metabolism.

\section{Inflammatory Cytokines}

The challenge with inflammatory cytokine assessments is that their production follows a circadian rhythm [58]; therefore, variation in assessment timing in research may explain the inconsistent findings described in the literature about the association between inflammation and comorbidities. Characteristically, BBB is a robust interface between peripheral tissues and the brain. Cytokines are large molecules that cannot penetrate BBB, and mostly work in a paracrine fashion. However, evidence suggests that systemic inflammation generates leaky regions in BBB which allow the entry of cytokines [59]. Additionally, the brain endothelial cytokine-transport molecules and activation of vagal afferent fibers transmit cytokine signals to specific brain nuclei, which add to the inflammatory response. Stereotypically, these fibers contain in their perineural sheath macrophages and dendritic cells that express membrane Toll-Like Receptors and produce IL-1 $\beta$ when activated [60]. Cytokines are important part of the innate immunity that is crucial for a normal brain function. However, elevated levels of cytokines modify the neurocircuitry and neurotransmitter systems; consequently, behavioral alterations lead to mental distress and feeding problems. Mechanisms of cytokine inducedbehavioral changes involve variations in monoamine, glutamate, and neuropeptide systems and reduction in growth factors namely the brain-derived neurotrophic factor. Evidence suggests that tryptophan metabolism in the CNS is modified by inflammatory cytokines and high cortisol levels to produce a range of toxic kynurenine metabolites by the glial cells. In neuroinflammation over $98 \%$ of the kynurenine formed in the brain is derived from local production [61]. Some excitotoxic metabolites activate the $\mathrm{N}$-methyl-D-aspartate (NMDA)-type glutamate receptors and increase oxidative stress as well as neuronal damage. In fact, kynurenine metabolism has been related to several human behavior disorders [62]. Additionally, peripheral kynurenine competes with tryptophan and other LNAA transport to the brain adding to the etiology of lower central 5-HT production [63].

Insulin resistance ensues from a systemic inflammation and is a potential contributor to altered feeding behaviors and mental distress [64,65]. Insulin receptors have been localized in areas of the brain crucial to food intake regulation and energy homeostasis [66]. However, unlike leptin, insulin is thought to play a feebler effect on feeding. Pharmacological interventions that promote insulin sensitivity such as Metformin reduce food intake and promote weight loss in animal and human models. Metformin's action is mediated through inhibition of NPY and AgRP neurons in the hypothalamus $[67,68]$. Taken all together, systemic inflammation and chronic stress reduce brain serotonin levels by shifting tryptophan metabolism into toxic metabolites that further disrupt brain circuitry. Insulin resistance adds to the deranged communication in the brain by increasing hunger and promoting mental distress.

\section{Leptin and Serotonin: What's the Link with Mental Health?}

Leptin exerts its anorexic effects through multiple mechanisms. Leptin modulates the effect of Krüppel-like factor 4 KLF-4 (a zinc finger transcription factor) which typically activates AgRP neurons [69]. Leptin stimulates POMC neurons to reduce feeding [52] and moderates brain and peripheral serotonin levels [70]. However, the intricate functions of leptin make studying feeding behaviors challenging. In animal models, leptin administration increases serum serotonin levels and reduces hypothalamic and hippocampal serotonin concentrations. This inhibitory effect on serotonin synthesis mechanistically regulates appetite. Hypothalamic serotonin signaling inhibits up-regulation of key genes needed to 
induce satiety [71]. Therefore, selective targeting of serotonin in the hypothalamus might be a viable solution to treat hyperphagic diseases. The hypothalamic 5-HT2C receptors regulate energy balance and modulate glucose homeostasis [72]. Particularly, hypothalamic 5-HT2C and 5-HT1B receptors control POMC neurons. Therefore, a comprehensive approach that targets hypothalamic 5-HT2C may enhance leptin and insulin sensitivity. Reducing dietinduced inflammation appears to be key in enhancing metabolism, mood and feeding behaviors $[3,29]$.

\section{Conclusion}

The evidence provided in this review suggests that addressing mental distress and altered feeding behaviors requires a multistage approach. Dietary interventions require healthy ratios of protein to complex carbohydrates and of omega- 6 to omega- 3 fats. Additionally, regular cardiovascular exercise is warranted to improve leptin and insulin sensitivities and increase central 5-HT levels. Exercise also reduces stress which supports the multi-actions of leptin. During exercise, feeding is halted which may contribute to an intermittent rise in ghrelin. The slight increase in ghrelin levels supports its antistress function: Exercise also promotes fatigue and improves sleep which may increase Tph2 expression to boost brain 5-HT levels. Based on work from our lab, mood improvement will likely support motivation to improve diet and increase exercise frequency [40]. These proposed steps may become eventually a virtuous cycle that supports further stress reduction and improvement in central 5-HT. This approach could be used as an alternative medicine therapy or in conjunction with a pharmaceutical agent.

\section{References}

1. Fitzpatrick AL, Kuller LH, Lopez OL, Diehr P, O’Meara ES, et al. (2009) Midlife and late-life obesity and the risk of dementia: Cardiovascular health study. Arch Neurol 66(3): 336-342.

2. Ozawa M, Shipley M, Kivimaki M, Singh Manoux A, Brunner EJ (2016) Dietary pattern, inflammation and cognitive decline: The whitehall II prospective cohort study. Clin Nutr 36(2): 506-512.

3. De Git KC, Adan RA (2015) Leptin resistance in diet-induced obesity: The role of hypothalamic inflammation. Obes Rev 16(3): 207-224.

4. Raison CL, Capuron L, Miller AH (2006) Cytokines sing the blues: Inflammation and the pathogenesis of depression. Trends Immunol 27(1): 24-31.

5. Salim S, Chugh G, Asghar M (2012) Inflammation in anxiety. Adv Protein Chem Struct Biol 88: 1-25.

6. Khaodhiar L, Ling P, Blackburn GL, Bistrian BR (2004) Serum levels of interleukin- 6 and C-reactive protein correlate with body mass index across the broad range of obesity. JPEN J Parenter Enteral Nutr 28(6): 410-415.

7. Hanada T, Yoshimura A (2002) Regulation of cytokine signaling and inflammation. Cytokine Growth Factor Rev 13(4-5): 413-421.

8. Gershon MD, Tack J (2007) The serotonin signaling system: From basic understanding to drug development for functional GI disorders. Gastroenterology 132(1): 397-414.

9. Berger M, Gray JA, Roth BL (2009) The expanded biology of serotonin. Annu Rev Med 60(1): 355-366.
10. Nautiyal KM, Hen R (2017) Serotonin receptors in depression: From A to B. F1000 Res 6: 123.

11. Mengod G, Vilaró M, Cortés R, López Giménez J, Raurich A, et al. (2006) Chemical neuroanatomy of 5-HT receptor subtypes in the mammalian brain. The serotonin receptors pp. 319-364.

12. Araneda R, Andrade R (1991) 5-hydroxytryptamine 2 and 5 -hydroxytryptamine $1 \mathrm{~A}$ receptors mediate opposing responses on membrane excitability in rat association cortex. Neuroscience 40(2): 399-412.

13. Jennings J, Ung R, Resendez S, Stamatakis A, Taylor J, et al. (2015) Visualizing hypothalamic network dynamics for appetitive and consummatory behaviors. Cell 160(3): 516-527.

14. Carter ME, Soden ME, Zweifel LS, Palmiter RD (2013) Genetic identification of a neural circuit that suppresses appetite. Nature 503(7474): 111-114.

15. Feijó Fde M, Bertoluci MC, Reis C (2011) Serotonin and hypothalamic control of hunger: A review. Rev Assoc Med Bras (1992) 57(1): 74-77.

16. Lin S, Lee L, Yang YK (2014) Serotonin and mental disorders: A concise review on molecular neuroimaging evidence. Clin Psychopharmacol Neurosci 12(3): 196-202.

17. Juruena MF, Cleare AJ, Pariante CM (2004) The hypothalamic pituitary adrenal axis, glucocorticoid receptor function and relevance to depression. Rev Bras Psiquiatr 26(3): 189-201.

18. Herman JP, McKlveen JM, Ghosal S, Kopp B, Wulsin A, et al. (2016) Regulation of the hypothalamic-pituitary-adrenocortical stress response. Compr Physiol 6(2): 603-621.

19. Linkowski P, Mendlewicz J, Kerkhofs M, Leclercq R, Golstein J, et al. (1987) 24-hour profiles of adrenocorticotropin, cortisol, and growth hormone in major depressive illness: effect of antidepressant treatment. J Clin Endocrinol Metab 65(1): 141-152.

20. Zhang X, Beaulieu JM, Sotnikova TD, Gainetdinov RR, Caron MG (2004) Tryptophan hydroxylase-2 controls brain serotonin synthesis. Science 305(5681): 217.

21. Zill P, Büttner A, Eisenmenger W, Bondy B, Ackenheil M (2004) Regional mRNA expression of a second tryptophan hydroxylase isoform in postmortem tissue samples of two human brains. Eur Neuropsychopharmacol 14(4): 282-284

22. Chen G, Miller GM (2012) Advances in tryptophan hydroxylase-2 gene expression regulation: New insights into serotonin-stress interaction and clinical implications. Am J Med Genet B Neuropsychiatr Genet 159B (2): 152-171.

23. Malek ZS, Sage D, Pévet P, Raison S (2007) Daily rhythm of tryptophan hydroxylase-2 messenger ribonucleic acid within raphe neurons is induced by corticoid daily surge and modulated by enhanced locomotor activity. Endocrinology 148(11): 5165-5172.

24. Alenina N, Kikic D, Todiras M, Mosienko V, Qadri F, et al. (2009) Growth retardation and altered autonomic control in mice lacking brain serotonin. Proc Natl Acad Sci U S A 106(25): 10332-10337.

25. Tecott LH, Sun LM, Akana SF, Strack AM (1995) Eating disorder and epilepsy in mice lacking 5-HT2c serotonin receptors. Nature 374(6522): 542-546.

26. Heisler LK, Zhou L, Bajwa P, Hsu J, Tecott LH (2007) Serotonin 5-HT(2C) receptors regulate anxiety-like behavior. Genes Brain Behav 6(5): 491496.

27. Giorgetti M, Tecott LH (2004) Contributions of 5-HT2C receptors to multiple actions of central serotonin systems. Eur J Pharmacol 488(13): $1-9$. 
28. Wurtman RJ, Fernstrom JD (1976) Control of brain neurotransmitter synthesis by precursor availability and nutritional state. Biochem Pharmacol 25(15): 1691-1696.

29. Chen L, Chen R, Wang H, Liang F (2015) Mechanisms linking inflammation to insulin resistance. Int J Endocrinol 2015(2015): 1-9.

30. Lawson EA, Miller KK, Blum JI, Meenaghan E, Misra M, et al. (2012) Leptin levels are associated with decreased depressive symptoms in women across the weight spectrum, independent of body fat. Clin Endocrinol 76(4): 520-525.

31. Lin P, Huang S, Su K (2010) A meta-analytic review of polyunsaturated fatty acid compositions in patients with depression. Biol Psychiatry 68(2): 140-147.

32. Simopoulos AP (2016) An increase in the omega-6/omega-3 fatty acid ratio increases the risk for obesity. Nutrients 8(3): 128.

33. Mocking RJT, Ruhé HG, Assies J, Lok A, Koeter MWJ, et al. (2013) Relationship between the hypothalamic-pituitary-adrenal-axis and fatty acid metabolism in recurrent depression. Psychoneuroendocrinology 38(9): 1607-1617.

34. Grosso G, Galvano F, Marventano S, Malaguarnera M, Bucolo C, et al (2014) Omega-3 fatty acids and depression: Scientific evidence and biological mechanisms. Oxid Med Cell Longev 2014: 313570.

35. Jiang L, Liang Q, Shi Y (2012) Pure docosahexaenoic acid can improve depression behaviors and affect HPA axis in mice. Eur Rev Med Pharmacol Sci 16(13): 1765-1773.

36. Gray B, Steyn F, Davies PSW, Vitetta L (2013) Omega-3 fatty acids: A review of the effects on adiponectin and leptin and potential implications for obesity management. Eur J Clin Nutr 67(12): 1234-1242.

37. Abella V, Scotece M, Conde J, Pino J, Gonzalez Gay MA, et al. (2017) Leptin in the interplay of inflammation, metabolism and immune system disorders. Nat Rev Rheumatol 13(2): 100-109.

38. Kasperek GJ, Dohm GL, Snider RD (1985) Activation of branched-chain keto acid dehydrogenase by exercise. Am J Physiol 248(2): 166-171.

39. Dey S, Dey PK, Singh RH (1992) Exercise training: Significance of regional alterations in serotonin metabolism of rat brain in relation to antidepressant effect of exercise. Physiol Behav 52(6): 1095-1099.

40. Begdache L, Chaar M, Sabounchi N, Kianmehr H (2017) Assessment of dietary factors, dietary practices and exercise on mental distress in young adults versus matured adults: A cross-sectional study. Nutr Neurosci 11: 1-11.

41. Perry B, Wang Y (2011) Appetite regulation and weight control: The role of gut hormones. Nutr Diabetes 2: e26.

42. Da Silva Pereira JA, Da Silva F, De Moraes Vieira PM (2017) The impact of ghrelin in metabolic diseases: An immune perspective. J Diabetes Res 2017: 4527980 .

43. Yin Y, Li Y, Zhang W (2014) The growth hormone secretagogue receptor: Its intracellular signaling and regulation. Int J Mol Sci 15(3): 4837-4855.

44. Kim E, Grover LM, Bertolotti D, Green TL (2010) Growth hormone rescues hippocampal synaptic function after sleep deprivation. Am J Physiol Regul Integr Comp Physiol 298(6): 1588-1596.

45. Spencer SJ, Xu L, Clarke MA, Lemus M, Reichenbach A, et al. (2012) Ghrelin regulates the hypothalamic-pituitary-adrenal axis and restricts anxiety after acute stress. Biol Psychiatry 72(6): 457-465.

46. Smiley DL, Tschöp M, Heiman ML (2000) Ghrelin induces adiposity in rodents. Nature 407(6806): 908-913.

47. Perez Tilve D, Heppner K, Kirchner H, Lockie SH, Woods SC, et al. (2011) (2011) Ghrelin-induced adiposity is independent of orexigenic effects. FASEB J 25(8): 2814-2822.
48. Baver SB, Hope K, Guyot S, Bjørbaek C, Kaczorowski C, et al. (2014) Leptin modulates the intrinsic excitability of AgRP/NPY neurons in the arcuate nucleus of the hypothalamus. J Neurosci 34(16): 5486-5496.

49. Caro JF, Kolaczynski JW, Nyce MR, Ohannesian JP, Opentanova I, et al. (1996) Decreased cerebrospinal-fluid/serum leptin ratio in obesity: A possible mechanism for leptin resistance. Lancet 348(9021): 159-161.

50. Ur E, Wilkinson DA, Morash BA, Wilkinson M (2002) Leptin immunoreactivity islocalized toneurons in ratbrain. Neuroendocrinology 75(4): 264-272.

51. Müller MJ, Bosy Westphal A, Heymsfield SB (2010) Is there evidence for a set point that regulates human body weight? F1000 Med Rep 2: 59.

52. Dodd GT, Decherf S, Loh K, Simonds S, Wiede F, et al. (2015) Leptin and insulin act on POMC neurons to promote the browning of white fat. Cell 160(1-2): 88-104.

53. Wauman J, Zabeau L, Tavernier J (2017) The leptin receptor complex: Heavier than expected? Front Endocrinol 8: 30.

54. Pinto S, Roseberry AG, Liu H, Diano S, Shanabrough M, et al. (2004) Rapid rewiring of arcuate nucleus feeding circuits by leptin. Science 304(5667): 110-115

55. Sohn JW (2015) Network of hypothalamic neurons that control appetite. BMB Rep 48(4): 229-233.

56. Bornstein SR, Uhlmann K, Haidan A, Ehrhart Bornstein M, Scherbaum WA (1997) Evidence for a novel peripheral action of leptin as a metabolic signal to the adrenal gland: Leptin inhibits cortisol release directly. Diabetes 46(7): 1235-1238.

57. Lu XY, Kim CS, Frazer A, Zhang W (2006) Leptin: A potential novel antidepressant. Proc Natl Acad Sci U S A 103(5): 1593-1598.

58. Arvidson NG, Gudbjoernsson B, Elfman L, Ryden A, Toetterman TH, et al. (1994) Circadian rhythm of serum interleukin-6 in rheumatoid arthritis. Ann Rheum Dis 53(8): 521-524.

59. Varatharaj A, Galea I (2016) The blood-brain barrier in systemic inflammation. Brain Behav Immun 60: 1-12.

60. Dantzer R (2009) Cytokine, sickness behavior, and depression. Immunol Allergy Clin North Am 29(2): 247-264.

61. Kita T, Morrison PF, Heyes MP, Markey SP (2002) Effects of systemic and central nervous system localized inflammation on the contributions of metabolic precursors to the l-kynurenine and quinolinic acid pools in brain J Neurochem 82(2): 258-268.

62. Haroon E, Raison CL, Miller AH (2012) Psychoneuroimmunology meets neuropsychopharmacology: Translational implications of the impact of inflammation on behavior. Neuropsychopharmacology 37(1): 137-162.

63. Fukui S, Schwarcz R, Rapoport SI, Takada Y, Smith QR (1991) Bloodbrain barrier transport of kynurenines: Implications for brain synthesis and metabolism. J Neurochem 56(6): 2007-2017.

64. Scinta W, Bayes H, Smith N (2017) Insulin resistance and hunger in childhood obesity: A patient and physician's perspective. Adv Ther 34(10): 2386-2391.

65. Lawlor DA, Smith GD, Ebrahim S (2003) Association of insulin resistance with depression: Cross sectional findings from the british women's heart and health study. BMJ 327(7428): 1383-1384.

66. Abizaid A, Horvath TL (2008) Brain circuits regulating energy homeostasis. Regul Pept 149(1-3): 3-10.

67. Ye W, Ramos EH, Wong BC, Belsham DD (2016) Beneficial effects of metformin and/or salicylate on palmitate- or TNF $\alpha$-induced neuroinflammatory marker and neuropeptide gene regulation in immortalized NPY/AgRP neurons. Plos One 11(11): e0166973. 
68. Lee A, Morley JE (1998) Metformin decreases food consumption and induces weight loss in subjects with obesity with type II non-insulindependent diabetes. Obes Res 6(1): 47-53.

69. Imbernon M, Sanchez Rebordelo E, Gallego R, Gandara M, Lea P, et al. (2014) Hypothalamic KLF4 mediates leptin's effects on food intake via AgRP. Mol Metab 3(4): 441-451.

70. Haleem DJ, Haque Z, Inam Q Ikram H, Haleem MA (2015) Behavioral, hormonal and central serotonin modulating effects of injected leptin Peptides 74: 1-8.
71. Yadav VK, Oury F, Tanaka K, Thomas T, Wang Y, et al. (2011) Leptindependent serotonin control of appetite: Temporal specificity, transcriptional regulation, and therapeutic implications. J Exp Med 208(1): 41-52.

72. Tecott LH, Abdallah L (2003) Mouse genetic approaches to feeding regulation: Serotonin 5-HT2C receptor mutant mice. CNS Spectr 8(8): 578-588.
Creative Commons Attribution 4.0 International License

For possible submissions Click Here

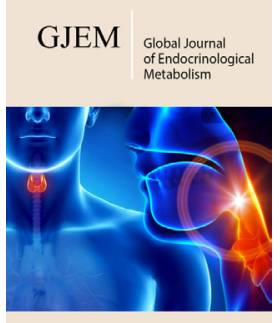

Global Journal of Endocrinological Metabolism

\section{Benefits of Publishing with us}

- High-level peer review and editorial services

- Freely accessible online immediately upon publication

- Authors retain the copyright to their work

- Licensing it under a Creative Commons license

- Visibility through different online platforms 